\title{
Bacterial cellulose production on whey - an overview of prospects
}

\section{Sergejs Koḷesovs, Kristaps Neiberts, Maija Rukliša, Pāvels Semjonovs*}

Institute of Biology, University of Latvia, Ojāra Vācieša street 4, Riga, Latvia, LV-1004

*Corresponding author: psem@latnet.lv

Keywords: whey, bacterial cellulose, acetic acid bacteria, whey valorisation

Bacterial cellulose (BC) is a biopolymer with a wide range of potential applications starting from the food and packaging industry to biomedicine and electronics. Despite its high potential, BC large-scale production remains still challenging (Jozala et al., 2015; Azeredo et al., 2019). The high cost of growth media, which can reach up to $30 \%$ of production costs is one of them. To decrease production costs, the use of industrial and agricultural by-products (Table 2), including whey (Table 1 and 2), as alternative growth media can be considered. Whey is the main high-volume by-product of the dairy industry and can be considered as an alternative growth medium for BC production despite its low valorisation opportunities. Only a few research articles are dedicated to the evaluation of BC productivity on whey media. BC production on whey medium is highly strain-specific and is associated with strains' ability to hydrolyse lactose which is the main C source in whey (Semjonovs et al., 2017; Revin et al., 2018). Several strains do not support BC synthesis on whey or lactose-containing media (Thompson and Hamilton, 2001).

Our study shows that Komagataeibacter rhaeticus P-1463 can produce up to $1.95 \pm 0.15 \mathrm{~g} / \mathrm{L}$ of BC (dry weight) during 10 days of cultivation on the unhydrolyzed whey medium, thus assimilating lactose and relevant monosaccharides as $\mathrm{C}$ substrate. Moreover, K. rhaeticus $\mathrm{P}-1463$ utilises galactose and lactose in a modified HS medium (Table 1). Whey enzymatic pretreatment with $\beta$-galactosidase increased BC production by K. rhaeticus P-1463 to $2.41 \mathrm{~g} / \mathrm{L}$ (dry weight). Probably there are limiting factors besides $\mathrm{C}$ and $\mathrm{N}$ concentrations restricting $\mathrm{BC}$ production in whey medium. 
Table 1. BC production on standard and modified HS media (K. rhaeticus P-1463)

\begin{tabular}{|l|c|c|}
\hline \multicolumn{1}{|c|}{ Medium } & BC dry weight, g/L & Productivity QX, g/L/d \\
\hline HS medium & $3.68 \pm 0.03$ & 0.37 \\
\hline Modified HS medium with lactose & $2.85 \pm 0.04$ & 0.29 \\
\hline Modified HS medium with galactose & $2.1 \pm 0.02$ & 0.21 \\
\hline Whey & $1.95 \pm 0.15 \mathrm{~g} / \mathrm{L}$ & 0.19 \\
\hline
\end{tabular}

Table 2. Comparison of $B C$ production on whey with other alternative substrates.

Table adapted from (Kolesovs and Semjonovs, 2020)

\begin{tabular}{|c|c|c|c|}
\hline Mudium & Strain & BC dry weight, $g / L$ & Reference \\
\hline $\begin{array}{l}\text { Whey medium (no } \\
\text { pre-treatment) }\end{array}$ & K. rhaeticus P-1463 & 1.95 & Current research \\
\hline $\begin{array}{l}\text { Hydrolised whey } \\
\text { ( } \beta \text {-galactosidase } \\
\text { pre-treated) }\end{array}$ & K. rhaeticus P-1463 & 2.41 & Current research \\
\hline Whey & $\begin{array}{c}\text { G. sucrofermentans } \\
\text { B-11267 }\end{array}$ & 5.45 & (Revin et al., 2018) \\
\hline Whey & K. xylinus DSM 2325 & 6.77 & (Rollini et al., 2020) \\
\hline Corn steep liqour & $\begin{array}{c}\text { A. xylinum NRRL } \\
\text { B-42 }\end{array}$ & 6.7 & $\begin{array}{c}\text { (Cerrutti et al., } \\
\text { 2016) }\end{array}$ \\
\hline Orange juice & $\begin{array}{c}\text { A. xylinum NBRC } \\
13693\end{array}$ & 5.9 & $\begin{array}{c}\text { (Kurosumi et al., } \\
\text { 2009) }\end{array}$ \\
\hline Apple juice & $\begin{array}{c}\text { A. xylinum NBRC } \\
13693\end{array}$ & 3.9 & $\begin{array}{c}\text { (Kurosumi et al., } \\
\text { 2009) }\end{array}$ \\
\hline Pineaple juice & $\begin{array}{c}\text { A. xylinum NBRC } \\
13693\end{array}$ & 3.9 & $\begin{array}{c}\text { (Kurosumi et al., } \\
\text { 2009) }\end{array}$ \\
\hline Molases & A. xylinum BPR 2001 & 5.3 & $\begin{array}{c}\text { (Bae and Shoda, } \\
\text { 2004) }\end{array}$ \\
\hline Glycerol & $\begin{array}{c}\text { G. xylinus CGMCC } \\
\text { no. } 2955\end{array}$ & 5.97 & (Zhong et al., 2013) \\
\hline $\begin{array}{l}\text { Wheat enzymatic } \\
\text { hydrolysate }\end{array}$ & $\begin{array}{c}\text { A. xylinus ATCC } \\
23770\end{array}$ & 8.3 & (Chen et al., 2013) \\
\hline $\begin{array}{l}\text { Coconut water (Nata } \\
\text { de Coco) }\end{array}$ & Acetic acid bacteria & 712.9 (wet weight) & (Phong et al., 2017) \\
\hline
\end{tabular}




\section{Conclusions}

Currently whey can be recognised as challenging and still quite a problematic alternative growth substrate for large-scale $\mathrm{BC}$ production. It was shown that whey is a suitable and promising medium for $\mathrm{BC}$ synthesis by acetic acid bacteria strain $K$. rhaeticus $\mathrm{P}-1463$. At the same time, the use of untreated whey results in significantly lower BC if compared to standard HS medium. Further extensive studies may improve the prospects in both - the search for cheap alternative growth substrates for industrial BC production and valorisation of whey.

\section{Acknowledgments}

Project No. 19-00-A01612-000004 "Obtaining of bio-degradable polymers from renewable resources for the production of protective coatings and packaging materials for fruits" is co-financed by the European agricultural fund for rural development (EAFRD). This study was supported by the Ministry of Agriculture and Rural Support Service of the Republic of Latvia.

\section{References}

Azeredo, H. M. C., Barud, H., Farinas, C. S., Vasconcellos, V. M., and Claro, A. M. 2019. Bacterial Cellulose as a Raw Material for Food and Food Packaging Applications. Front Sustain Food Syst 3. https://doi.org/10.3389/fsufs.2019.00007

Bae, S., Shoda, M. 2004. Bacterial cellulose production by fed-batch fermentation in molasses medium. Biotechnol Prog. 20: 1366-1371. https://doi.org/10.1021/bp0498490

Cerrutti, P., Roldán, P., García, R. M., Galvagno, M. A., Vázquez, A., and Foresti, M. L. 2016. Production of bacterial nanocellulose from wine industry residues: Importance of fermentation time on pellicle characteristics. J Appl Polym Sci. 133: 1-9. https://doi.org/10.1002/app.43109

Chen, L., Hong, F., Yang, X.-xia, and Han, S.-fen 2013. Biotransformation of wheat straw to bacterial cellulose and its mechanism. Bioresour Technol. 135: 464-468. https://doi.org/10.1016/j. biortech.2012.10.029

Jozala, A. F., Pértile, R. A. N., dos Santos, C. A., de Carvalho Santos-Ebinuma, V., Seckler, M. M., Gama, F. M., and Pessoa, A. 2015. Bacterial cellulose production by Gluconacetobacter xylinus by employing alternative culture media. Appl Microbiol Biotechnol. 99: 1181-1190. https://doi.org/ $10.1007 /$ s00253-014-6232-3

Kolesovs, S., Semjonovs, P. 2020. Production of bacterial cellulose from whey -current state and prospects. Appl Microbiol Biotechnol. 104: 7723-7730. https://doi.org/10.1007/s00253-02010803-9

Kurosumi, A., Sasaki, C., Yamashita, Y., and Nakamura, Y. 2009. Utilization of various fruit juices as carbon source for production of bacterial cellulose by Acetobacter xylinum NBRC 13693. Carbohydr Polym. 76: 333-335. https://doi.org/10.1016/j.carbpol.2008.11.009 
Phong, H. X., Lin, L. T., Thanh, N. N., Long, B. H., and Dung, N. T. P. 2017. Investigating the conditions for nata-de-coco production by newly isolated Acetobacter sp. Am J Food Sci Nutr. 4: 1-6.

Revin, V., Liyaskina, E., Nazarkina, M., Bogatyreva, A., and Shchankin, M. 2018. Cost-effective production of bacterial cellulose using acidic food industry by-products. Brazilian J Microbiol. 49: 151-159. https://doi.org/10.1016/j.bjm.2017.12.012

Rollini, M., Musatti, A., Cavicchioli, D., Bussini, D., Farris, S., Rovera, C., Romano, D., De Benedetti, S., and Barbiroli, A. 2020. From cheese whey permeate to Sakacin-A/bacterial cellulose nanocrystal conjugates for antimicrobial food packaging applications: a circular economy case study. Sci Rep. 10: 1-14. https://doi.org/10.1038/s41598-020-78430-y

Semjonovs, P., Ruklisha, M., Paegle, L., Saka, M., Treimane, R., Skute, M., Rozenberga, L., Vikele, L., Sabovics, M., and Cleenwerck, I. 2017. Cellulose synthesis by Komagataeibacter rhaeticus strain P 1463 isolated from Kombucha. Appl Microbiol Biotechnol. 101(3): 1003-1012.

Thompson, D. N., Hamilton, M. A. 2001. Production of bacterial cellulose from alternate feedstocks. Appl Biochem Biotechnol - Part A Enzym Eng Biotechnol. 91-93: 503-513. https://doi.org/10.1385/ ABAB:91-93:1-9:503

Zhong, C., Zhang, G. C., Liu, M., Zheng, X. T., Han, P. P., and Jia, S. R. 2013. Metabolic flux analysis of Gluconacetobacter xylinus for bacterial cellulose production. Appl Microbiol Biotechnol. 97: 6189-6199. https://doi.org/10.1007/s00253-013-4908-8 